\title{
\%.
}

\section{Über die Divisoren gewisser Formen der Zahlen, welche aus der Theorie der Kreistheilung. entstehen.}

(Von Herrn Dr. E. E. Kummer, Professor an der Universität zu Breslau.)

Wenn man die imaginären Wurzeln der Gleichung $x^{p}=1$, in welcher $p$ eine Primzahl ist, in Perioden theilt, und zwar in $e$ Perioden von je $f$ Gliedern, wenn $p-1=e \cdot f$ ist, so sind diese bekanntlich die Wurzeln einer algebraischen Gleichung eten Grades, in welcher der Coëfficient des höchsten Gliedes gleich Eins ist und alle übrigen ganze Zahlen sind. Ist $x$ irgend eine imaginäre Wurzel der Gleichung $x^{p}=1$ und $g$ eine primitive Wurzel für die Primzahl $p$, so haben diese Perioden, welche kurz durch $\eta, \eta_{1}, \eta_{2}, \ldots \eta_{e-1}$ bezeichnet werden sollen, folgende Werthe:

$$
\begin{aligned}
& \eta=x+x^{g^{e}}+x^{g^{2 e}}+\ldots+x^{g(f-1) e}, \\
& \eta_{1}=x^{g}+x^{o e+1}+x^{g^{2 e+1}}+\ldots+x^{g}{ }^{(f-1 e+1}, \\
& \eta_{i-1}=x^{g^{e-1}}+x^{g^{2 e-1}}+x^{g^{3 e-1}}+\ldots+x^{g^{f_{e-1}}} \text {. }
\end{aligned}
$$

Setzl man nun

$$
\varphi(y)=(y-\eta)\left(y-\eta_{1}\right)\left(y-\eta_{2}\right) \ldots\left(y-\eta_{e-1}\right),
$$

so hat man auch

$$
\varphi(y)=y^{e}+a_{1} y^{e-1}+a_{2} y^{e-2}+\ldots+a_{e-1} y+a_{e},
$$

wo $a_{1}, a_{2}, a_{3}, \ldots a_{e}$ ganze Zahlen sind; welche in jedem speciellen Fall bestimmt werden können. Wir werden nun diese ganze rationale Function $\varphi(y)$ vom eten Grade als Form betrachten, unter welcher gewisse Zahlen dargestellt werden können, und namentlich die Divisoren untersuchen, welche diese Form haben kann. $\mathrm{Zu}$ diesem $\mathrm{Zwecke}$ werden wir Congruenzen anwenden, in welchen nicht nur reale ganze Zahlen, sondern auch die irrationalen, oft imaginären Perioden $\eta, \eta_{1}, \eta_{2}, \ldots \eta_{e}$ vorkommen, und darum müssen wir zunächst den Sinn festhalten, welchen solche Congruenzen haben sollen. Bekanntlich läfst sich jede ganze rationale Function dieser Perioden, welche ganze Zahlen zu Coëfficienten hat, auf die lineäre Form $c \eta+c_{1} \eta_{1}+c_{2} \eta_{2}+\ldots$ $\ldots+c_{\mathrm{e}-1} \eta_{e-1}$ bringen, und zwar nur auf eine einzige $W$ eise. Wir wollen also 
den Begriff der Congruenz für den gegenwärtigen Zweck dahin ausdehnen, dafs zwei ganze rationale Functionen der Perioden für den Modul $q$, welcher eine ganze Zahl sein soll, congruent heifsen sollen, wenn in dem Unterschiede derselben, nachdem er auf die Form $c \eta+c_{1} \eta_{1}+\ldots+c_{e-1} \eta_{e-1}$ gebracht worden ist, alle Coëfficienten $c, c_{1}, c_{2}, \ldots c_{e-1}$ durch $q$ theilbar sind. Nach dieser Erklärung kann man hier, ebenso wie bei den gewöhnlichen Congruenzen, alle Glieder, welche den Modul $q$ als Factor enthalten, weglassen; auch kann man diese Congruenzen ebenso mit einander addiren, subtrahiren, multipliciren und zu Potenzen erheben.

Wir gehen nun von dem bekannten Satze aus, dafs, wenn q eine Primzahl ist, die Coëfficienten $b, b_{1}, b_{2}, \ldots b_{q-1}$ des entwickelten Products

$$
z(z-1)(z-2) \ldots(z-q+1)=z^{q}-b_{1} z^{q-1}+b_{2} z^{q-2}-\ldots+b_{q-1} z
$$

alle durch $q$ theilbar sind, mit Ausnahme des letzten $b_{q-1}$, welcher durch $q$ dividirt den Rest -1 läfst. Es ist nemlich für jeden Werth des $z$ dieses Product, als Product von $q$ auf einander folgenden ganzen Zahlen, durch $q$ theilbar, also ist auch

$$
z^{q}-b_{1} z^{q-1}+b_{2} z^{q-2}-\ldots+b_{q-1} z \equiv 0 \text { Mod. } q,
$$

und da nach dem Fermatschen Satze $z^{q} \equiv z$ ist, so ist auch

$$
-b_{1} z^{q-1}+b_{2} z^{q-2}-\ldots+\left(b_{q-1}+1\right) z \equiv 0 \quad \text { Mod. } q \text {. }
$$

Diese Congruenz vom Grade $q-1$ kann aber nicht $q$ verschiedene Wurzeln haben: also mufs sie identisch erfüllt werden, woraus $b_{1} \equiv b_{2} \equiv b_{3} \equiv \ldots$. $\ldots \equiv b_{q-2} \equiv b_{q-1}+1$ Mod. $q$ folgt.

Nimmt man nun in der obigen Gleichung $z=y-\eta_{k}$ und läfst die durch $q$ theilbaren Glieder weg, so erhält man die Congruenz

$$
\begin{gathered}
\left(y-\eta_{k}\right)\left(y-1-\eta_{k}\right)\left(y-2-\eta_{k}\right) \ldots\left(y-q+1-\eta_{k}\right) \\
\equiv\left(y-\eta_{k}\right)^{q}-\left(y-\eta_{k}\right) \quad \text { Mod. } q .
\end{gathered}
$$

Bekanntlich sind aber in der binomischen Entwickelung einer qten Potenz, wenn $q$ eine Primzahl ist, alle Glieder, mit Ausnahme des ersten und letzten, durch $q$ theilbar, also ist $\left(y-\eta_{k}\right) \equiv y^{q}-\eta_{k}^{q}$. Ferner, ist $\eta_{k}$ ein Polynom von $f$ Gliedern, und wenn ein solches zur $q$ ten Potenz erhoben wird, so sind die Coëfficienten aller Glieder durch $q$ theilbar, mit Ausnahme der qten Potenzen der einzelnen $f$ Glieder, also ist

$$
\eta_{k}^{q} \equiv x^{q g^{k}}+x^{q g^{e+k}}+x^{q g^{2 c+k}}+\ldots+x^{q g^{(f-1) e+k}} \quad \text { Mod. } q,
$$

und wenn $q \equiv g^{r}$ Mod. $p$ ist, so hat man hiernach

$$
\eta_{k}^{q} \equiv \eta_{r+k} \text { Mod. } q \text {, }
$$


in dem besondern Falle aber, wo $q=p$ ist,

$$
\eta_{k}^{p} \equiv f \text { Mod. } p \text {. }
$$

Die Congruenz $\left(y-\eta_{k}\right)^{q} \equiv y^{q}-\eta_{k}^{q}$ Mod. $q$ geht also allgemein in $\left(y-\eta_{k}\right)^{q}$ $\equiv y-\eta_{k+r}$ Mod. $q$ über, und für den besondern Fall $q=p$ giebt sie $\left(y-\eta_{k}\right)^{p} \equiv y-f$ Mod. $p$.

Giebt man nun hierin dem $k$ nach einander die Werthe $0,1,2, \ldots e-1$ und bildet das Product, so erhält man

$$
(\varphi(y))^{p} \equiv(y-f)^{e} \quad \text { Mod. } p \text {; }
$$

woraus folgt, dafs $\varphi(y)$ für $y \equiv f$ den Factor $p$ hat, aber für keinen andern Werth des $y$; oder dafs die Congruenz $\varphi(y) \equiv 0$ Mod. $p$ stets eine reelle Wurzel hat, nemlich $y=f=\frac{p-1}{e}$.

Wir kehren nun zur Untersuchung des allgemeinen Primfactors $q$ zurück, für welchen $q \equiv g^{r}$ Mod. $p$ ist. Vermöge der Congruenz $\left(y-\eta_{k}\right)^{q} \equiv y-\eta_{k+r}$ verwandelt sich die Congruenz (A.) in folgende:

(B.) $\left(y-\eta_{k}\right)\left(y-1-\eta_{k}\right)\left(y-2-\eta_{k}\right) . \ldots\left(y-q+1-\eta_{k}\right) \equiv \eta_{k}-\eta_{k+r}$ Mod. q. Es sind die beiden Fälle besonders zu betrachten: erstens wo $r$ durch $e$ theilbar ist, und zweitens, wo dies nicht der Fall ist. Ist $r$ durch $e$ theilbar, so ist $\eta_{k}=\eta_{k+r}$, also

$$
\left(y-\eta_{k}\right)\left(y-1-\eta_{k}\right)\left(y-2-\eta_{k}\right) \ldots\left(y-q+1-\eta_{k}\right) \equiv 0 \quad \text { Mod. } q .
$$

Setzt man nun nach einander $k=0,1,2, \ldots e-1$ und bildet das Product aller dieser Congruenzen, so erhält man

$$
\varphi(y) \varphi(y-1) \varphi(y-2) \ldots \varphi(y-q+1) \equiv 0 \quad \text { Mod. } q^{e} .
$$

Es müssen also immer $\theta$ dieser Factoren durch $q$ theilbar sein, oder auch einige derselben den Factor $q$ mehreremal enthalten, wenn $q \equiv g^{r}$ Mod. $p$, und $\boldsymbol{r}$ durch $e$ theilbar ist, dafs heifst, wenn $q$ ein eter Potenzrest der Primzahl $q$ ist. Hieraus erhält man folgenden Lehrsatz:

"Jede Primzahl, welche ein eter Potenzrest von $p$ ist, ist ein Divisor „ der Form $\varphi(y)$; oder auch so: die Congruenz $\varphi(y) \equiv 0$ Mod. $q$ hat, „wenn der Modul $q$ eine Primzahl und zugleich eter Potenzrest von $p$ „ist, immer $e$ reelle Wurzeln, welche in besondern Fällen auch zum "Theil einander gleich werden können."

Der besondere Fall, wo die Perioden eingliedrig, also die imaginären Wurzeln der Gleichung $x^{p}=1$ selbst sind, giebt das bekannte Resultat, dafs die Congruenz $x^{p-1}+x^{p-2}+x^{p-3}+\ldots+x+1 \equiv 0$ Mod. $q$ stets $p-1$ reelle Wur- 
zeln hat, wenn die Primzahl $q$ ein $p-1$ ter Potenzrest von $p$ ist, d. h. wenn $q=2 m p+1$ ist.

Nachdem gezeigt worden, dafs alle diejenigen Primzablen, welche ete Potenzreste von $p$ sind, Divisoren der Form $\varphi(y)$ sind, ist zweitens zu untersuchen, ob diese Form, aufser diesen genannten und dem Divisor $p$, noch andere Divisoren haben kann, oder nicht. Es sei also wieder $q \equiv g^{r}$ Mod. $p$, aber $r$ nicht durch $e$ theilbar. In diesem Falle giebt die Congruenz $(\boldsymbol{B}$.$) , wenn nach einander k=0,1,2, \ldots e-1$ gesetzt und das Product gebildet wird:

$$
\begin{aligned}
& \varphi(\boldsymbol{y}) \varphi(\boldsymbol{y}-1) \varphi(y-2) \ldots \varphi(y-\boldsymbol{y}+1) \equiv \boldsymbol{P} \text { Mod. } \boldsymbol{\varphi}, \\
& \boldsymbol{P}=\left(\eta-\eta_{r}\right)\left(\eta_{1}-\eta_{r+1}\right)\left(\eta_{2}-\eta_{r+2}\right) \ldots\left(\eta_{e-1}-\eta_{r+e-1}\right) \text { ist. }
\end{aligned}
$$

wo

$\boldsymbol{P}$, als symmetrische Function aller Perioden, ist eine ganze Zahl. Für bestimmte $\boldsymbol{p}$ und $e$ kann diese Zahl, auch wenn man alle verschiedenen Werthe des $\boldsymbol{r}$ zuläfst, nur eine endliche, bestimmte und verhältnifsmäfsig sehr geringe Anzahl verschiedener Primfactoren enthalten, und da $\varphi(y)$ keine andern Primfactoren der genannten Art enthalten kann, als diejenigen, welche auch in $\boldsymbol{P}$ vorkommen, so folgt, dafs $\varphi(y)$ nur ausnahmsweise eine stets begrenzte Anzahl solcher Primfactoren enthalten kann, welche nicht ete Potenzreste von $p$ sind. Um näher zu untersuchen, in welchen Fällen dergleichen ausnahmsweise Primfactoren des $\boldsymbol{P}$, und somit auch des $\varphi^{\prime}(\boldsymbol{y})$, Statt haben können, gebrauchen wir die Congruenz

$$
\left(\eta_{k}-\eta_{r+k}\right)^{q} \equiv \eta_{k+r}-\eta_{k+2 r} \quad \text { Mod. } q \text {, }
$$

deren Richtigkeit nach den oben aufgestellten Principien in die Augen fällt. Werden beide Seiten dieser Congruenz zu wiederholten Malen zur qten Potenz erhoben, so erhält man die allgemeinere Congruenz

$$
\text { (C.) } \quad\left(\eta_{k}-\eta_{r+k}\right)^{q^{h}} \equiv \eta_{h r+k}-\eta_{(h+1) r+k} \text { Mod. q. }
$$

Macht man hierin nacheinander $\iota=0,1,2, \ldots e-1$ und bildet das Product, so erhălt man

$$
\left(\eta_{k}-\eta_{r+k}\right)^{1+q+q^{2}+\ldots+\varphi^{e-1}} \equiv\left(\eta_{k}-\eta_{r+k}\right)\left(\eta_{r+k}-\eta_{2 r+k}\right) \ldots\left(\eta_{(e-1) r+k}-\eta_{e r+k}\right) \text { Mod. q. }
$$

Wenn nun $r$ keinen gemeinschaftlichen Factor mit $e$ hat, so sind die Indices der Perioden $k, r+k, 2 r+k, \ldots(e-1) r+k$, in anderer Ordnung genommen, den Indices $0,1,2, \ldots e-1$ congruent, für den Modul $e$; das Product rechterhand ist also kein anderes als das Product $\boldsymbol{P}$, und da $\boldsymbol{P}$ nach der Voraussetzung durch $q$ theilbar sein soll, so hat man

$$
\left(\eta_{k}-\eta_{r+i k}\right)^{1+q+q^{2}+\ldots .+q^{e-1}} \equiv 0 \text { Mod. } q \text {. }
$$


Wenn nun zur Potenz $q-1$ erhoben wird, so ist

$$
\left(\eta_{k}-\eta_{r+k}\right)^{q^{e}-1} \equiv 0 \text { Mod. } q \text {, }
$$

und, wenn mit $\eta_{k}-\eta_{r+k}$ multiplicirt wird,

$$
\left(\eta_{k}-\eta_{k+r}\right)^{q^{e}} \equiv 0 \text { Mod. q, }
$$

woraus nach der Congruenz (C.) folgt:

$$
\eta_{k}-\eta_{r+k} \equiv 0 \text { Mod. } q \text {; }
$$

welches unmöglich ist. Das Product $\boldsymbol{P}$ hat also keinen Primfactor $q$ von der Art, dafs $q \equiv g^{r}$ Mod. $p$, wo $r$ keinen gemeinschaftlichen Factor mit $e$ hat. Für den Fall also, wo $e$ Primzahl ist, hat man folgenden Lehrsatz:

„Die Form $\varphi(y)$ hat, wenn der Grad derselben $e$ eine Primzahl ist, aufser "dem Divisor $\boldsymbol{p}$ nur solche Divisoren, welche ete Potenzreste von $\boldsymbol{p}$ sind." Für den Fall aber, wo der Grad der Form $\varphi(y)$ keine Primzahl ist, kann man das gefundene Resultat folgendermaafsen aussprechen.

„Die Form $\varphi(y)$ hat aufser dem Divisor $p$ im allgemeinen nur solche „Primzahlen zu Divisoren, welche ete Potenzreste von $p$ sind; aufserdem „aber kann sie auch eine endliche bestimmte Anzahl anderer Divisoren "haben, welche, wenn $e$ die von Eins verschiedenen Divisoren $\alpha, \beta, \gamma, \ldots$ "enthält, $\alpha$ te oder $\beta$ te oder $\gamma$ te Potenzreste von $p$ sein müssen."

Man kann die Bedingungen, unter welchen $\varphi(y)$ solche besondere Divisoren enthält, die nicht ete Potenzreste sind, noch etwas genauer angeben. Wenn nemlich $\boldsymbol{r}$ und $e$ den gröfsten gemeinschaftlichen Factor $\alpha$ enthalten, und $\boldsymbol{r}=\boldsymbol{r}^{\prime} \alpha$, $e=e^{\prime} \alpha$ ist, so setze man in der Congruenz $\left(C\right.$.) nach einander $h=0,1,2, \ldots e^{\prime}-1$ und bilde das Product der so erhaltenen Congruenzen:

$$
\left.\eta_{k}-\eta_{r+k}\right)^{1+q+q^{2}+\ldots+q^{e^{\prime}-1}} \equiv\left(\eta_{k}-\eta_{r+k}\right)\left(\eta_{r+k}-2 r+k\right) \ldots\left(\eta_{\left(e^{\prime}-1\right) r+k}-\eta_{e^{\prime} r+k}\right) .
$$

Giebt man hierin wieder dem $k$ die Werthe $0,1,2, \ldots \alpha-1$ und bildet das Product, so wird dieses Product auf der rechten Seite gleich $\boldsymbol{P}$; wovon man sich sogleich überzeugt, wenn man bemerkt, dafs die Zahlen von der Form $h r+k$ für $h=0,1,2, \ldots e^{\prime}-1$ und $k=0,1,2, \ldots \alpha-1$, wenn $r=r^{\prime} \alpha$ und $e=e \alpha$, für den Modul $e$ alle Reste $0,1,2,3, \ldots e-1$ geben. Man hat also, da $\boldsymbol{P}$ durch $\boldsymbol{q}$ theilbar sein soll,

$$
\left\{\left(\eta-\eta_{r}\right)\left(\eta_{1}-\eta_{r+1}\right) \ldots\left(\eta_{\alpha-1}-\eta_{\alpha+r-1}\right)\right\}^{1+q+q^{2}+\ldots .+q^{e^{\prime}-1}} \equiv 0 \text {. Mod. q. }
$$

Erhebt man wieder zur Potenz $q-1$ und multiplicirt mit $\left(\eta-\eta_{r}\right)\left(\eta_{1}-\eta_{r+1}\right) \ldots$ $\ldots\left(\eta_{\alpha-1}-\eta_{\alpha+r-1}\right)$, so folgt hieraus

$$
\left\{\left(\eta-\eta_{r}\right)\left(\eta_{1}-\eta_{r+1}\right) \ldots\left(\eta_{\alpha+1}-\eta_{\alpha+r-1}\right)\right\}^{q^{e^{\prime}}} \equiv 0 \text { Mod. } q \text {, }
$$


welches vermöge der Congruenz $(\boldsymbol{C}$.) folgende einfachere Form annimmt:

$$
\left(\eta-\eta_{r}\right)\left(\eta_{1}-\eta_{r+1}\right) \ldots\left(\eta_{\alpha-1}-\eta_{\alpha+r-1}\right) \equiv 0 \quad \text { Mod. } q \text {. }
$$

Es müssen also schon die ersten $\alpha$ Factoren des Products $\boldsymbol{P}$ den Factor $q$ enthalten, damit $\boldsymbol{P}$ oder $\varphi(y)$ denselben enthalten könne. Man könnte durch diese einschränkende Bedingung auf die Vermuthung kommen, dafs diese ausnahmsweisen Factoren, welche nicht ete Potenzreste sind, auch wenn $e$ eine zusammengesetzte Zahl ist, überhaupt gar nicht vorkommen dürften: dafs dieses indefs nicht der Fall ist, vielmehr wirklich dergleichen Factoren Statt haben, kann man an folgendem einfachen Beispiele sehen. Nimmt man $p=109$, $e=6$, so erhält man nach bekannten Methoden:

$$
\varphi(y)=y^{6}+y^{5}-45 y^{4}-10 y^{3}+135 y^{2}+9 y-27,
$$

woraus sich leicht für $y=0,1,2,3,4,5$ folgende Werthe des $\varphi(y)$ berechnen lassen: $\varphi(0)=-3^{3} ; \varphi(1)=2^{6} ; \varphi(2)=-173 ; \varphi(3)=-2^{6} \cdot 3^{3} ; \varphi(4)=$ $-4871 ; \varphi(5)=-2^{6} \cdot 113$. Die hierin vorkommenden Divisoren 2 und 3 sind keine 6ten Potenzreste von 109; dieselben sind also solche ausnahmsweise Divisoren; und es ist 2 ein cubischer Rest und 3 quadratischer Rest von 109; welches sehr wohl mit dem oben gefundenen Lehrsatze stimmt. Übrigens sind 2 und 3 im gegenwärtigen Falle die einzigen Primfactoren von $\varphi(y)$, welche nicht sechste Potenzreste sind.

Wir zeigen nun noch von zwei wichtigen speciellen Fällen, dafs für sie dergleichen Factoren, welche nicht ete Potenzreste von $\boldsymbol{p}$ sind, niemals Statt haben können: nemlich für den Fall, wo die Perioden eingliedrig, und wo sie zweigliedrig sind. Ist $e=p-1$ und $f=1$, so ist $\eta=x, \eta_{1}=x^{g}$, $\eta_{2}=x^{g^{2}}$ etc.; es ist also

$$
\boldsymbol{P}=\left(x-x^{g}\right)\left(x^{g}-x^{g^{2}}\right) \ldots\left(x^{g p-z}-x^{g^{p-1}}\right),
$$

und wenn man von dem ersten Factor $x$, vom zweiten $x^{g}$, vom dritten $x^{g^{2}}$ etc., heraushebt, so wird

$$
\boldsymbol{P}=\left(1-x^{g-1}\right)\left(1-x^{g(g-1}\right) \ldots\left(1-x^{g-2}(g-1)\right),
$$

und da

$$
\left(z-x^{m}\right)\left(z-x^{m g}\right) \ldots\left(z-x^{m \cdot p^{p-2}}\right)=z^{p-1}+z^{p-2}+\ldots z+1
$$

ist, so hat man, wenn $z=1$ und $m=g-1$ genommen wird,

$$
\boldsymbol{P}=\boldsymbol{p} \text {. }
$$

Da nun $\boldsymbol{P}$ keinen andern Factor enthält als $\boldsymbol{p}$, so folgt hieraus der bekannte Lehrsatz: dafs die Form $y^{p-1}+y^{p-2}+\ldots+y+1$ aufser dem Divi- 
sor $p$ nur solche Divisoren enthalten kann, welche $p-1$ te Potenzreste von $p$ sind, deren lineäre Form also $q=2 m p+1$ ist.

Wenn ferner die Perioden zweigliedrig sind, also $e=\frac{1}{2}(p-1), f=2$ ist, so ist $\eta=x+x^{-1}, \eta_{1}=x^{g}+x^{-g}, \eta_{2}=x^{g^{2}}+x^{-g^{2}}$ etc., und es wird für diesen Fall bekanntlich

$\varphi(y)=y^{e}+y^{e-1}-\frac{e-1}{1} y^{e-2}-\frac{e-2}{1} y^{e-3}+\frac{(e-2)(e-3)}{1 \cdot 2} y^{e-4}+\frac{(e-3)(e-4)}{1 \cdot 2} y^{e-5}-\ldots$

Ferner ist

$$
\eta_{k}-\eta_{r+k}=x^{g^{k}}+x^{-g^{k}}-x^{g^{r+k}}-x^{-g^{r+k}},
$$

welcher Ausdruck folgendermaafsen in Factoren zerfällt:

$$
\eta_{k}-\eta_{r+k}=x^{g^{k}}\left(1-x^{\left(g^{r}-1\right) g^{k}}\right)\left(1-x^{-\left(g^{r}+1\right) g^{k}}\right) \text {. }
$$

Giebt man nun dem $k$ die Werthe $0,1,2, \ldots p-2$ und bildet das Product, so erhält man leicht

$$
\boldsymbol{P}^{2}=\boldsymbol{p}^{2}, \quad \text { also } \boldsymbol{P}= \pm p \text {. }
$$

In diesem Falle hat also $\boldsymbol{P}$ ebenfalls keinen andern Divisor als $\boldsymbol{p}$, woraus folgt, dafs die Form

$$
\varphi(y)=y^{e}+y^{e-1}-\frac{e-1}{1} y^{e-2}-\frac{e-2}{1} y^{e-3}+\ldots
$$

aufser dem Divisor $p$ nur solche Divisoren hat, welche $\frac{1}{2}(p-1)$ te Potenzreste von $p$ sind, also von der Form $2 m p \pm 1$.

Dieselbe Methode, welche in dem Vorhergehenden für die Form $\varphi(y)$ angewendet wurde, giebt fast ganz in derselben Weise die Primfactoren einer weit allgemeineren, sehr merkwürdigen Form vom eten Grade mit $e$ unbestimmten Zahlen. Wenn man nemlich folgende zusammengehörige lineäre Functionen der Perioden:

$$
\begin{aligned}
& \boldsymbol{F}(\eta)=z \eta+z_{1} \eta_{1}+z_{2} \eta_{2}+\ldots .+z_{e-1} \eta_{e-1}, \\
& \boldsymbol{F}\left(\eta_{1}\right)=z \eta_{1}+z_{1} \eta_{2}+z_{2} \eta_{3}+\ldots+z_{e-1} \eta \text {, } \\
& \boldsymbol{F}\left(\eta_{e-1}\right)=z \eta_{e-1}+z_{1} \eta+z_{2} \eta_{1}+\ldots+z_{e-1} \eta_{e-2}
\end{aligned}
$$

mit einander multiplicirt, so wird das Product derselben

$$
\Psi=\boldsymbol{F}(\eta) \boldsymbol{F}\left(\eta_{1}\right) \boldsymbol{F}\left(\eta_{2}\right) \ldots \boldsymbol{F}\left(\eta_{e-1}\right)
$$

eine homogene Form vom eten Grade der $e$ unbestimmten Zahlen $z, z_{1}, z_{2}, \ldots$ $z_{e-1}$, mit ganzzahligen Coëfficienten; welche Form, wie wir sogleich zeigen Crelle's Journal f. d. M. Bd. XXX. Heft 2. 
werden, in Beziehung auf ihre Divisoren ganz mit der oben untersuchten specielleren Form $\varphi(y)$ übereinstimmt. Wir beweisen zunächst folgenden Lehrsatz: „Jede Primzahl $q$, welche ein eter. Potenzrest von $p$ ist, so wie auch $p$ "selbst, ist ein Divisor der Form $\Psi$."

Untersuchen wir zuerst den Divisor $p$, so haben wir, nach denselben Principien, welche oben angewendet wurden,

$$
\boldsymbol{F}\left(\eta_{k}\right)^{p} \equiv\left(z+z_{1}+z_{2}+\ldots+z_{e-1}\right) f . \text { Mod } p,
$$

also, wenn die unbestimmten Zahlen $z, z_{1}, z_{2}, \ldots z_{c-1}$ so bestimmt werden, dafs ihre Summe durch $\boldsymbol{p}$ theilbar ist, so wird $\boldsymbol{F}\left(\eta_{k}\right)^{p} \equiv 0$ Mod. $p$, und wenn man $k=0,1,2, \ldots e-1$ setzt und diese Congruenzen mit einander multiplicirt, so erhält man $\Psi^{p} \equiv 0$. Mod. $p$, also auch $\Psi \equiv 0$ Mod. $p ; p$ ist also ein Divisor der Form $\Psi$.

Um weiter zu beweisen, dafs auch jede Primzahl q, welche ein eter Potenzrest von $\boldsymbol{p}$ ist, immer Divisor von $\Psi$ sei, setze ich die zweite Periode $\eta$ als ganze rationale Function der ersten Periode $\eta$ dargestellt; welches bekanntlich immer möglich ist. Es sei also $\eta_{1}=\Theta(\eta)$, so ist $\eta_{2}=\Theta \Theta(\eta)$, $\eta_{3}=\Theta \Theta \Theta(\eta)$ etc. Statt $\eta$ aber werde eine unbestimmte Grölse $y$ genommen und folgender Ausdruck gebildet:

$$
\boldsymbol{F}(\boldsymbol{y}) \cdot \boldsymbol{F}(\Theta y) \cdot \boldsymbol{F}(\Theta \Theta y) \ldots \boldsymbol{F}\left(\Theta^{(e-1)} y\right)-\Psi,
$$

welcher eine ganze rationale Function von $y$ sein wird. Derselbe verschwindet offenbar, wenn $y=\eta$ oder $y=\eta_{1}$ oder $y=\eta_{2}$ etc. genommen wird, und mufs deshalb den Factor $(y-\eta)\left(y-\eta_{1}\right)\left(y-\eta_{2}\right) \ldots\left(y-\eta_{e-1}\right)$ enthalten, welchen wir oben durch $\varphi^{\prime}(y)$ bezeichnet haben. Es ist demnach

$$
\boldsymbol{F}(\boldsymbol{y}) \boldsymbol{F}(\Theta y)(\boldsymbol{F} \Theta \Theta y) \ldots \boldsymbol{F}\left(\Theta^{(e-1)} y\right)-\Psi=\varphi(y) \cdot \boldsymbol{\Phi}
$$

wo auch $\Phi$ eine ganze rationale Function von $y$ bedeutet. Wird nun für $y$ irgend eine Wurzel der Congruenz $\varphi(y) \equiv 0$ Mod. $q$ genommen (welche, wenn $q$ ein eter Potenzrest von $p$ ist, immer $e$ reale Wurzeln hat, wie wir oben gezeigt haben), so hat man:

$$
\boldsymbol{F}(\boldsymbol{y}) \boldsymbol{F}(\Theta y) \boldsymbol{F}(\Theta \Theta y) \ldots \boldsymbol{F}\left(\Theta^{(e-1)} y\right) \equiv \Psi \quad \text { Mod. } \boldsymbol{q} .
$$

Wenn also irgend ein Factor dieses Productes durch $q$ theilbar wird, so wird allemal auch $\Psi$ durch $q$ theilbar. Es lassen sich daher die unbestimmten Zahlen $z, z_{1}, z_{2}, \ldots z_{c-1}$ immer so bestimmen, dafs die Form $\Psi$ den Divisor $q$ erhält, und die Bestimmung derselben ist einfach die, dafs, wenn man in

$$
\boldsymbol{F}(\eta)=z \eta+z_{1} \eta_{1}+z_{2} \eta_{2}+\ldots .+z_{e-1} \eta_{e-1}
$$

für $\eta, \eta_{1}, \eta_{2}, \ldots \eta_{e-1}$ nicht mehr die Wurzeln der Gleichung $\varphi(g)=0$, 
sondern die Wurzeln der Congruenz $\varphi(y) \equiv 0$ Mod. $q$, aber in der gehörigen Ordnung genommen, substituirt hat, $F(\eta) \equiv 0$ Mod. $q$ werden mufs.

Die Primfactoren, welche ete Potenzreste von $p$ sind, machen auch hier die hauptsächlichsten Divisoren der Form $\Psi$ aus, und aufser diesen haben gewisse andere nur ausnahmsweise Statt, deren Bedingungen wir jetzt näher untersuchen wollen. Es sei $q$ eine Primzahl, von der Art, dafs $q \equiv g^{r}$ Mod. $p$ und $r$ nicht durch $e$ theilbar ist, so ist

$$
\boldsymbol{F}(\eta)^{q} \equiv \boldsymbol{F}^{\prime}\left(\eta_{r}\right) \quad \text { Mod. } q .
$$

Erhebt man dies nun zu wiederholten Malen zur Potenz $q$, so erhält man

$$
\boldsymbol{F}(\eta)^{q^{h}} \equiv \boldsymbol{F}\left(\eta_{h r}\right) \quad \text { Mod. } \boldsymbol{q} \text {. }
$$

Setzt man nach einander $h=0,1,2,3, \ldots e-1$ und multiplicirt die so erhaltenen Congruenzen in einander, so wird

$$
\boldsymbol{F}(\eta)^{1+q+q^{2}+\ldots .+q^{e-1}} \equiv \boldsymbol{F}(\eta) \boldsymbol{F}\left(\eta_{r}\right) \boldsymbol{F}\left(\eta_{2 r}\right) \ldots \boldsymbol{F}\left(\eta_{(e-1) r}\right) \quad \text { Mod. } q .
$$

Wenn nun $r$ und $e$ keinen gemeinschaftlichen Factor haben, so sind die Indices $0, r, 2 r, 3 r, \ldots(e-1) r$ den Indices $0,1,2, \ldots e-1$, in anderer Ordnung genommen, congruent für den Modul $e$, also ist das Product rechterhand gleich $\Psi$. Soll nun $\Psi$ den Divisor $q$ enthalten, so folgt, dafs

$$
\boldsymbol{F}(\eta)^{1+q+q^{2}+\ldots .+q^{e-1}} \equiv 0 \quad \text { Mod. } q
$$

sein mufs, woraus, ebenso wie oben, $F(\eta) \equiv 0$ Mod. $q$ folgt. Damit aber $\boldsymbol{F}(\eta)$ durch $q$ theilbar sei, müssen alle einzelnen Coëfficienten der Perioden, also die Unbestimmten $z, z_{1}, z_{2}, \ldots z_{c-1}$, durch $q$ theilbar sein. Hieraus erhalten wir folgenden Lehrsatz:

"Wenn die unbestimmten Zahlen der Form $\Psi$ nicht alle einen gemein"schaftlichen Factor haben, so enthält diese Form aufser dem Divisor $p$ "nur solche Primfactoren, welche ete Potenzreste von $p$ sind; oder: wenn "e die von Eins verschiedenen Divisoren $\alpha, \beta, \gamma, \ldots$ enthält, so kann die "Form auch solche Primfactoren enthalten, welche $\alpha$ te oder $\beta$ te oder $\gamma$ te .... "Potenzreste von $p$ sind."

Daraus geht auch folgender Zusatz hervor:

"Wenn die unbestimmten Zahlen der Form $\Psi$ nicht alle einen gemeinschaft"lichen Factor enthalten, und der Grad $e$ dieser Form ist eine Primzahl, so „enthält dieselbe aufser dem Divisor $p$ nur solche Divisoren, welche ete "Potenzreste von $p$ sind."

Ist $e$ nicht eine Primzahl, sondern $\alpha$ ein Divisor von $e$, so sei $q \equiv g^{r}$ Mod. $p$ und $\alpha$ der gröfste gemeinschaftliche Factor von $r$ und $e$, so dafs $r \equiv r^{\prime} \alpha$ und 
$e=e^{\prime} \alpha . \quad$ In diesem Falle hat man, vermöge der Congruenz

$$
\boldsymbol{F}\left(\eta_{k}^{q^{h}}\right) \equiv \boldsymbol{F}\left(\eta_{r h+k}\right) \quad \text { Mod. } \boldsymbol{q}
$$

wenn nach einander $h=0,1,2, \ldots e^{\prime}-1$ gesetzt wird und sodann für jeden einzelnen dieser Werthe des $h$ die Zahl $k$ die Werthe $0,1,2, \ldots \alpha-1$ bekommt,

$$
\left\{\boldsymbol{F}(\eta) \boldsymbol{F}\left(\eta_{1}\right) \boldsymbol{F}\left(\eta_{2}\right) \ldots \boldsymbol{F}^{\prime}\left(\eta_{\alpha-1}\right)\right\}^{1+q+q^{2}+\ldots+q^{e^{\prime}-1}} \equiv \Psi \quad \text { Mod. } \boldsymbol{q} ;
$$

denn das Product rechter Hand wird offenbar gleich $\Psi$, weil $\boldsymbol{h r}+\boldsymbol{k}$ für die angegebenen Werthe des $h$ und $k$ allen den Zahlen $0,1,2, \ldots e-1$ congruent wird, für den Modul $e$. Soll nun $\Psi$ den Factor $q$ haben, so folgt, dafs $\left\{\boldsymbol{F}(\eta) \boldsymbol{F}\left(\eta_{1}\right) \ldots \boldsymbol{F}\left(\eta_{a-1}\right)\right\}^{1+q+q^{2}+\ldots .+q^{e^{\prime-1}}} \equiv 0 \quad$ Mod. $q$ sein mufs, woraus man leicht folgert, dafs auch

$$
\boldsymbol{F}(\eta) \boldsymbol{F}\left(\eta_{1}\right) \boldsymbol{F}\left(\eta_{2}\right) \ldots \boldsymbol{F}\left(\eta_{\alpha-1}\right) \equiv \mathbf{0} \quad \text { Mod. } q
$$

sein mufs. Damit also $\Psi$ einen Primfactor $q$ habe, welcher nicht eter Potenzrest, sondern nur ater Potenzrest von $p$ ist, wo $\alpha$ ein Divisor von $e$, mufs allemal schon das Product der ersten $\alpha$ Factoren des $\Psi$ diesen Factor $q$ enthalten. 